\title{
Comportamiento de la rugosidad superficial del acero luego de exposición natural en exterior e interior
}

\section{Behavior of superficial roughness of the steel after natural outdoor and indoor exposure}

\author{
Jeyris Martínez Gutiérrez; Emilio Miguel Lluveras Pérez²; Rigoberto Marrero Águila ${ }^{3}$
}

11Ing. Química. Laboratorio de Ensayos de Tropicalización, Carretera El Morro No. 7246 Km 2 1², Reparto Cojímar, La Habana, Cuba, e-mail: jeyris@labet.ctec. cu, (1Dhttps://orcid.org/0000-0002-0564-8661

${ }^{2}$ Ing. Mecánico. Laboratorio de Ensayos de Tropicalización, Carretera El Morro No. 7246 Km 2 1⁄2, Reparto Cojímar, La Habana, Cuba, e-mail: emilio@labet. ctec.cu, Dhttps://orcid.org/0000-0002-3648-6204

${ }^{3}$ Ing. Químico, Doctor en Ciencias Técnicas. Instituto Superior Politécnico "José Antonio Echeverría", ISPAJE, Avenida 114, Reparto Marianao, La Habana, Cuba, e-mail: rigo@quimica.cujae.edu.cu, Dhttps://orcid.org/0000-0002-3011-5701

Cómo citar: Martínez Gutiérrez, J.; Lluveras Pérez, E.M.; Marrero Águila, R. 2019. Comportamiento de la rugosidad superficial del acero luego de exposición natural en exterior e interior. Rev. U.D.C.A Act. \& Div. Cient. 22(1):e1193. https://doi.org/10.31910/rudca. v22.n1.2019.1193

Artículo de acceso abierto publicado por Revista U.D.C.A Actualidad \& Divulgación Científica bajo una licencia Creative Commons CC BY-NC 4.0

Recibido: Octubre 1 de 2018

Aceptado: Marzo 20 de 2019

\section{RESUMEN}

El conocimiento de los fenómenos que tienen lugar en el medio que nos rodea permite garantizar la preservación y el uso adecuado de los recursos, elevando la calidad de vida en el planeta, para el bienestar presente y futuro de los hombres que lo habitan. Uno de los procesos naturales a los que nos enfrentamos todos y cada uno de los seres que coexistimos en este planeta, sean vivos o no, es el deterioro, debido a la interacción con el medio ambiente. La forma más natural de deterioro en que los metales regresan a minerales, como se encuentran en la naturaleza, es conocida como corrosión, proceso que es afectado por múltiples factores ambientales. El estado superficial de una muestra metálica resulta en un aspecto que influye en la magnitud y tipo de corrosión. En el presente trabajo, se evalúo el comportamiento de la rugosidad superficial en condiciones de exposición natural en exteriores e interiores, durante un corto tiempo, de $360 \mathrm{~h}$. A partir del diseño multifactorial, se demuestra la incidencia de las condiciones de exposición y el tiempo de ensayo en la rugosidad superficial, con un nivel de confianza del 95\%. Las ecuaciones del modelo lineal describen la relación entre la rugosidad superficial y el tiempo de exposición, posibilitando posibles predicciones en el futuro, para condiciones del clima, similares a las descritas en el estudio.

Palabras clave: corrosión; rugosidad; estadística; acero (https:/ / www.thesaurus.com/browse/thesaurus).

\section{ABSTRACT}

Knowledge of the phenomena that take place in the environment that surrounds us, ensures the preservation and proper use of resources, raising the quality of life on the planet for the present and future well-being of the men who inhabit it. One of the natural processes that we face each and every one of the beings that coexist on this planet, whether alive or not, is the deterioration due to interaction with the environment. The most natural form of deterioration in which metals return to minerals, as they are found in nature, is known as corrosion, a process that is affected by multiple environmental factors. The surface condition of a metal sample is an aspect that influences the magnitude and type of corrosion. In the present work we evaluated the behavior of the surface roughness in conditions of natural exposure in exteriors and interiors during a 
short time of $360 \mathrm{~h}$. Based on the multifactorial design, the incidence of exposure conditions and the test time on surface roughness with a $95 \%$ confidence level are demonstrated. The equations of the linear model describe the relationship between surface roughness and exposure time, making possible predictions in the future for climate conditions similar to those described in the study.

Keywords: corrosion; roughness; statistics; steel (Taken from https://www.thesaurus.com/browse/thesaurus).

\section{INTRODUCCIÓN}

El conocimiento de los fenómenos que tienen lugar en el medio que nos rodea permite garantizar la preservación y el uso adecuado de los recursos, elevando la calidad de vida en el planeta, para el bienestar presente y futuro de los hombres que lo habitan (López Cabrera et al. 2006).

Resulta difícil concebir ramas de la producción o de los servicios que manipulen o procesen tal cantidad de sustancias, como la industria química. Ella opera con sólidos, líquidos y gases, de naturaleza orgánica e inorgánica e, inclusive, con materia viva. Muchas de estas sustancias producen deterioro en los materiales, debido a la presencia de una disminución del tiempo de vida útil y funcionalidad de los mismos (Camejo Giniebra et al. 2018).

Estudios realizados afirman que la humanidad se enfrenta a un grave problema causado por la contaminación ambiental, la corrosión de los metales, entre ellos, la corrosión atmosférica del acero, principalmente, en las zonas costeras. Muchas estructuras, se han construido con este metal, debido a su resistencia mecánica (Guerra et al. 2018). Este es un fenómeno tan cotidiano como potencialmente destructivo, consumidor de recursos (Castaño et al. 2007). Tejero Rivas et al. (2015) han demostrado que el costo total de las pérdidas de metales por corrosión es, por lo menos, 4-5\% del PIB de un país.

Muchos trabajos, se han realizado, hasta la fecha, para determinar el deterioro estructural que sufren algunos materiales de construcción, relacionado con los cambios físicos y físicos-químicos, debido a que tienden a alcanzar formas termodinámicamente más estables y que poseen propiedades distintas a las originales deseadas (Camejo Giniebra et al. 2018).

El proceso de corrosión de metales expuestos a la atmósfera es de tipo electroquímico; tiene lugar cuando la superficie se encuentra humedecida, debido a diferentes fenómenos meteorológicos, como la lluvia, la condensación de humedad en el ambiente y la niebla (Chico et al. 2000).

Según Sánchez Muñoz (2017) existen diferentes factores que inciden en dicho proceso de corrosión, entre los que se encuentran el diseño, la interacción del metal con el medio ambiente, el estado de la superficie y los mecanismos deterioro; sin embargo, Ahmad (2006) plantea que inciden otros factores, tales como la temperatura, la humedad, la proximidad al mar, las lluvias, las partículas de polvo y la contaminación generada por el hombre.
Domínguez Domínguez \& Castro (1987) exponen que el estado superficial de una muestra metálica resulta un aspecto que influye en la magnitud y el tipo de corrosión. La rugosidad presente favorecerá el mayor establecimiento de micropilas galvánicas sobre la superficie, disolviéndose normalmente con mayor rapidez las crestas, las cimas y las salientes. Una mayor rugosidad impide el establecimiento de capas protectoras y pasivas más perfectas presentando puntos más susceptibles al ataque de agentes activos, provocando la corrosión localizada. De forma general, es bien conocido que una superficie bien pulida sufre menor corrosión que una rugosa. Una de las razones es la menor relación entre área real/área geométrica. Una placa de $1 \mathrm{~cm}^{2}$ puede presentar un área real 10 veces mayor, a consecuencia de rugosidades y de porosidades superficiales. A medida que el pulido es más exhaustivo, el área real se va acercando a la geometría y como la velocidad de corrosión depende de la primera, disminuye.

Dentro de los procesos productivos de la industria, se encuentra el traslado y el almacenamiento de las materias primas, que garantiza la conservación de las propiedades físicas y químicas de los materiales antes de ser utilizados. El estudio del comportamiento de la rugosidad superficial del acero juega un papel fundamental en los procesos de protección contra la corrosión, debido al aumento de la superficie de contacto entre el metal y el medio electrolítico.

Un limitado número de estudios se ha realizado para investigar la influencia de la rugosidad superficial sobre la corrosión de los aceros (Liu et al. 1995; Sasaki \& Burstein, 1996; Hong \& Nagumo, 1997; Moayed et al. 2003; Nor Asma et al. 2011; Mok Lee et al. 2012).

Sasaki \& Burstein (1996) demuestran que el cambio en el potencial sugiere que la nucleación y el crecimiento de las picaduras sobre una superficie suave es más difícil que sobre una superficie rugosa.

Nor Asma et al. (2011) afirman que la velocidad de corrosión aumentó al incrementar la rugosidad superficial del acero, ya sea en condición de flujo estática o turbulenta. La velocidad de corrosión en régimen de flujo turbulento fue mayor que en condición sin flujo para todos los acabados superficiales estudiados. Sugieren que la rugosidad superficial incrementa la velocidad de corrosión por aumentos en el área superficial, dispuesta para las reacciones electroquímicas.

Mok Lee et al. (2012) mostraron que la corrosión está correlacionada con la profundidad de los valles o surcos presentes en la superficie; las picaduras fueron preferentemente alineadas a lo largo de las ranuras y sugirieron que los valles profundos son lugares favorables para la nucleación de picaduras.

Las irregularidades presentes en la superficie pueden ser obtenidas a partir de la determinación de la rugosidad superficial. Los valores de rugosidad media aritmética $\mathrm{Ra}$, se definen como la altura de un rectángulo, cuya área sea igual a la suma absoluta de las áreas delimitadas entre el perfil de rugosidad y la línea media, siendo la longitud de medición Lm (Rodríguez Grasso et al. 2008). 


$$
R_{a}=\frac{1}{L_{m}} \int_{0}^{L_{m}}|y| d x \quad \sum A_{s}=\sum A_{i} \quad A_{T}=\sum A_{s}+\sum A_{i}
$$

Donde:

As: Áreas superiores

Ai: Áreas inferiores

Ат: Áreas totales

El grado de deterioro de los materiales, se relaciona directamente con el tipo de atmósfera al que se encuentra expuesto, siendo los factores climáticos y los contaminantes los que determinan el tipo de productos de la corrosión que se forman en la superficie de los metales (Del Angel-Meraz et al. 2009).

A diferencia de la situación en ambientes exteriores, en los que tanto el depósito seco como el húmedo, contribuyen a la acumulación de contaminantes en la superficie metálica, en los ambientes interiores, tal acumulación se debe casi exclusivamente al depósito seco y es, continuamente, aditiva para muchas especies. Probablemente, la diferencia más importante entre los ambientes exteriores e interiores es que, en estos últimos, el tiempo de humectación o los ciclos de mojado y secado se reducen a cero. Aunque los efectos de la corrosión no son muy visibles, pueden acarrear serios problemas, en el caso de materiales muy sensibles y costosos (Castaño et al. 2007).

Teniendo en cuenta la influencia que tiene el medio en el estado superficial del acero y que no se hayan reportado estudios acerca del tema en Cuba, en el presente trabajo, se pretende determinar la relación existente entre los cambios en la rugosidad superficial del acero a tiempos cortos de exposición en condiciones de exposición en exterior e interior, cuando no existe aún una capa de productos de corrosión formada sobre la superficie.

\section{MATERIALES Y MÉTODOS}

El desarrollo experimental de este trabajo, se efectuó en la estación de ensayos naturales del Laboratorio de Ensayos de Tropicalización (LABET). En este capítulo, se describen los materiales, la metodología seguida en el trabajo experimental y las condiciones en las que se realizaron las pruebas de los sistemas estudiados.

Selección y montaje de muestras. Para llevar a cabo el estudio, se seleccionó la estación de ensayos naturales, ubicada en Cojímar, al este de La Habana. La misma, se caracteriza por poseer uno de los microclimas de mayor agresividad de la República de Cuba y del mundo, dado a los altos valores de salinidad, humedad relativa, radiación solar y temperatura (González Prada \& Marrero Águila, 2005).

La estación, se encuentra situada en una zona de elevada agresividad corrosiva C5 (ISO, 2012), la cual, presenta una atmósfera costera industrial, siendo una de las más agresivas para las estructuras en climas tropicales, debido al comportamiento mensual de los parámetros meteorológicos, la deposición de contaminantes atmosféricos y la velocidad de corrosión.
A partir de estudios realizados por Suárez Corrales et al. (2014), se demuestra la influencia del relieve costero, formado por arrecifes, que contribuye también a la superior agresividad de esta estación, ya que el efecto de los rompientes de las olas en la costa, da lugar a la formación de abundante aerosol marino, lo que unido al marcado efecto transportador de los vientos provenientes del sector norte, cuando tiene lugar la entrada de los frentes fríos y de los vientos alisios del noreste al este, produce una suma de factores influyentes en la corrosión de los metales y en la degradación de los otros materiales específicos de este lugar.

Las muestras utilizadas para el estudio fueron ubicadas un grupo al aire libre, en contacto directo con las condiciones naturales y contaminantes atmosféricos y un segundo grupo en el interior de una caseta, protegidas contra la precipitación y la radiación solar, pero manteniendo el flujo de aire del exterior.

Para la preparación de las probetas de ensayo, se ha partido de chapas de acero de construcción, laminado en caliente y de bajo contenido de carbono. Se utilizaron 10 probetas para cada condición de ensayo (exterior e interior). Las placas son rectangulares y presentaban las siguientes dimensiones: $0,15 \times 0,10 \times 0,001 \mathrm{~m}$, las cuales, fueron colocadas a una distancia de $150 \mathrm{~m}$ desde el mar, con una orientación hacia el norte de frente a la costa y expuestas por un tiempo total de 360 horas.

Antes de la exposición en la estación natural, las probetas fueron desengrasadas con disolvente orgánico. No presentaban manchas de óxido visible o productos de corrosión en su superficie. Posteriormente, se efectuó el decapado químico para la eliminación de los productos de corrosión, teniendo en cuenta el procedimiento descrito en la norma ISO:9226 (1992).

Determinación de la rugosidad superficial del acero. La rugosidad, se define como todas aquellas irregularidades que forman el relieve de la superficie real y que, convencionalmente, se definen dentro de una zona, en la que se eliminan las desviaciones o errores de forma y las ondulaciones (Rodríguez Grasso et al. 2008).

Su determinación, se basa en el método de la medición de la textura superficial y está definido como la media aritmética de los valores absolutos de las coordenadas de los puntos del perfil de rugosidad, en relación a la línea media, dentro de la longitud de medición. Teniendo en cuenta la metodología descrita en la norma de rugosidad NC 16-60 (ONN, 1981), se efectuaron las mediciones, empleando el rugosímetro Surface Roughness Tester Huatec, Modelo SRT-6210.

Procedimiento. Antes de la utilización del instrumento fue calibrado, siguiendo las indicaciones del fabricante y los patrones de rugosidad establecidos. Se selecciona la opción "CAL" y se ajusta el patrón, no debiendo existir inclinación entre el equipo y la superficie de soporte, que se deben ubicar en posición paralela.

Al finalizar la calibración, se selecciona el paso de la medición con la opción (CUT OFF) y se comienza el ensayo. Se realizaron 
determinaciones de la rugosidad durante la exposición de las probetas en el ensayo de exposición natural, en exteriores e interiores.

Análisis estadístico. Para la realización del análisis estadístico, se utilizó el software Statgraphics Centurion XVII, que permite la evaluación de los resultados obtenidos en el ensayo de la rugosidad superficial, a partir de un análisis multifactorial. Dicho programa permite comprobar la existencia de significación estadística en los resultados obtenidos, durante la exposición de las muestras, en condiciones de exposición natural, exterior e interior. Se pretende obtener los modelos que mejor ajusten, teniendo en cuenta el tiempo de exposición en la variable respuesta, según las condiciones de exposición.

\section{RESULTADOS Y DISCUSIÓN}

Determinación de la rugosidad superficial del acero. Las muestras fueron expuestas por un período de tiempo de 360 horas, siendo evaluadas periódicamente cada 120 horas. Se pudo evidenciar, a simple vista, el incremento de la presencia de productos de corrosión en la superficie de las probetas. La figura 1 muestra la rugosidad de la probeta de acero antes de comenzar los ensayos climáticos, obtenida con la ayuda de un microscopio, con aumento de la imagen de $20 x$.

Fueron realizadas un total de 240 mediciones, donde 120 corresponden a las probetas expuestas en exteriores y 120, a

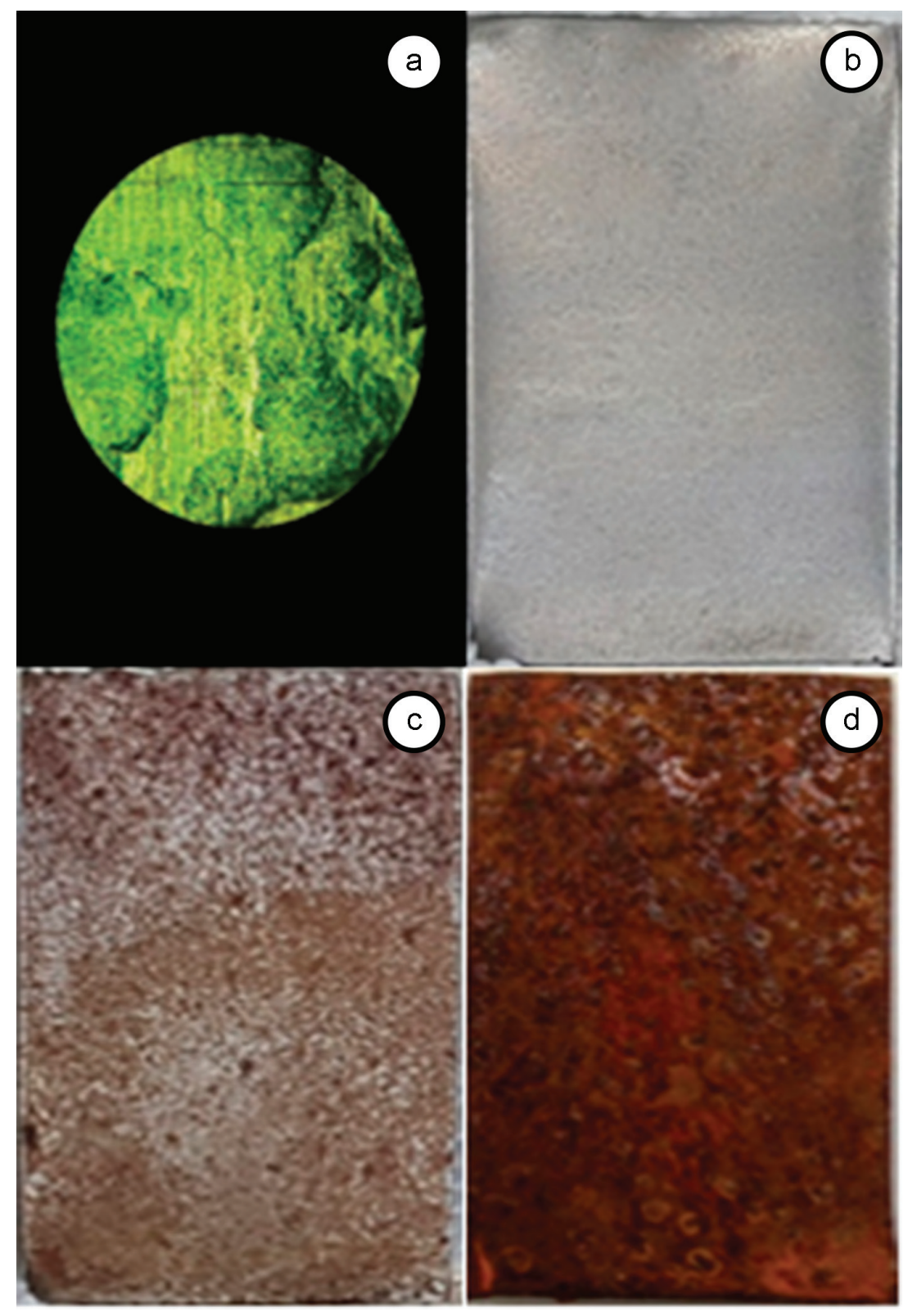

Figura 1. Imágenes obtenidas de las probetas de acero. a. Antes de ser ensayada con el microscopio aumento 20 X; b. Antes de ser ensayada; c. Después de 360 horas de exposición en el interior de la caseta; d. Después de 360 horas de exposición en el exterior. 
interiores. En cada tiempo de observación, se realizaron 30 mediciones, 3 por cada probeta. Los valores de rugosidad superficial obtenidos son reportados en las tablas 1 y 2.
En las probetas expuestas en el exterior, se evidenció el fenómeno de la corrosión, observándose la oxidación del metal, en diferentes zonas de la superficie metálica.

Tabla 1. Determinaciones de rugosidad superficial de las muestras ensayadas en condiciones de exposición exterior.

\begin{tabular}{|c|c|c|c|c|c|c|c|c|c|}
\hline \multirow{2}{*}{$\begin{array}{l}\text { Tiempo de } \\
\text { exposición } \\
\text { (h) }\end{array}$} & \multirow{2}{*}{ Identificación } & \multicolumn{3}{|c|}{$\begin{array}{c}\text { Mediciones de rugosidad } \\
\text { superficial } \mathrm{Ra}(\mu \mathrm{m})\end{array}$} & \multirow{2}{*}{$\begin{array}{l}\text { Promedio } \\
(\mu \mathrm{m})\end{array}$} & \multirow{2}{*}{$\begin{array}{c}\begin{array}{c}\text { Desviación } \\
\text { estándar } \\
(\mu \mathrm{m})\end{array}\end{array}$} & \multirow{2}{*}{$\begin{array}{c}\text { Coeficiente } \\
\text { de variación } \\
(\%)\end{array}$} & \multirow{2}{*}{$\begin{array}{c}\text { Sesgo } \\
\text { estandarizado }\end{array}$} & \multirow{2}{*}{$\begin{array}{c}\text { Curtosis } \\
\text { estandarizada }\end{array}$} \\
\hline & & $\begin{array}{c}\text { Medición } \\
1\end{array}$ & $\begin{array}{c}\text { Réplica } \\
1\end{array}$ & $\begin{array}{c}\text { Réplica } \\
2\end{array}$ & & & & & \\
\hline \multirow{10}{*}{0} & M 1-1 & 23,57 & 26,83 & 29,25 & \multirow{10}{*}{23,1987} & \multirow{10}{*}{4,1121} & \multirow{10}{*}{17,7258} & \multirow{10}{*}{0,0207} & \multirow{10}{*}{$-1,1495$} \\
\hline & M 1-2 & 27,76 & 29,44 & 29,62 & & & & & \\
\hline & M 1-3 & 22,91 & 21,33 & 23,66 & & & & & \\
\hline & M 1-4 & 23,01 & 25,53 & 19,75 & & & & & \\
\hline & M 1-5 & 18,84 & 16,81 & 17,29 & & & & & \\
\hline & M 1-6 & 22,29 & 19,67 & 17,41 & & & & & \\
\hline & M 1-7 & 22,89 & 18,00 & 16,21 & & & & & \\
\hline & M 1-8 & 27,06 & 23,01 & 27,75 & & & & & \\
\hline & M 1-9 & 20,62 & 21,82 & 29,57 & & & & & \\
\hline & M 1-10 & 22,89 & 27,09 & 24,08 & & & & & \\
\hline \multirow{10}{*}{120} & M 1-1 & 35,02 & 40,17 & 37,49 & \multirow{10}{*}{33,1640} & \multirow{10}{*}{5,8906} & \multirow{10}{*}{17,7620} & \multirow{10}{*}{0,2791} & \multirow{10}{*}{$-0,9395$} \\
\hline & M 1-2 & 29,66 & 28,84 & 32,96 & & & & & \\
\hline & M 1-3 & 44,08 & 40,79 & 39,82 & & & & & \\
\hline & M 1-4 & 32,91 & 32,26 & 32,39 & & & & & \\
\hline & M 1-5 & 24,99 & 21,68 & 24,87 & & & & & \\
\hline & M 1-6 & 30,22 & 27,93 & 27,42 & & & & & \\
\hline & M 1-7 & 36,89 & 35,45 & 35,84 & & & & & \\
\hline & M 1-8 & 29,71 & 26,78 & 26,65 & & & & & \\
\hline & M 1-9 & 32,14 & 31,50 & 31,50 & & & & & \\
\hline & M 1-10 & 41,30 & 41,83 & 41,83 & & & & & \\
\hline \multirow{10}{*}{240} & M 1-1 & 59,16 & 52,65 & 56,23 & \multirow{10}{*}{45,4587} & \multirow{10}{*}{11,9455} & & & \\
\hline & M 1-2 & 39,23 & 36,92 & 38,65 & & & & & \\
\hline & M 1-3 & 45,73 & 57,48 & 69,65 & & & & & \\
\hline & M 1-4 & 44,05 & 33,14 & 31,25 & & & & & \\
\hline & M 1-5 & 43,00 & 29,79 & 25,17 & & & 07ר & 16504 & 01010 \\
\hline & M 1-6 & 41,74 & 73,21 & 43,00 & & & $26,2 / 18$ & 1,0504 & 0,1910 \\
\hline & M 1-7 & 44,05 & 43,21 & 41,25 & & & & & \\
\hline & M 1-8 & 40,90 & 32,51 & 35,03 & & & & & \\
\hline & M 1-9 & 38,18 & 44,89 & 46,57 & & & & & \\
\hline & M 1-10 & 53,49 & 69,23 & 54,40 & & & & & \\
\hline & M 1-1 & 64,50 & 63,40 & 62,58 & & & & & \\
\hline & M 1-2 & 49,13 & 62,58 & 39,25 & & & & & \\
\hline & M 1-3 & 75,21 & 68,22 & 64,15 & & & & & \\
\hline & M 1-4 & 39,52 & 28,40 & 40,89 & & & & & \\
\hline 360 & M 1-5 & 29,59 & 30,19 & 37,99 & 523070 & 143700 & 260505 & 06412 & 14072 \\
\hline 360 & M 1-6 & 40,62 & 38,98 & 37,05 & $53,30 / 0$ & $14,3 / 08$ & 26,9585 & $-0,0412$ & $-1,49 / 3$ \\
\hline & M 1-7 & 39,25 & 64,50 & 64,82 & & & & & \\
\hline & M 1-8 & 72,44 & 71,00 & 69,00 & & & & & \\
\hline & M 1-9 & 54,33 & 54,66 & 52,67 & & & & & \\
\hline & M 1-10 & 56,24 & 62,28 & 65,77 & & & & & \\
\hline
\end{tabular}


Tabla 2. Determinaciones de rugosidad superficial de las muestras ensayadas en condiciones de exposición interior.

\begin{tabular}{|c|c|c|c|c|c|c|c|c|c|}
\hline \multirow{2}{*}{$\begin{array}{l}\text { Tiempo de } \\
\text { exposición } \\
\text { (h) }\end{array}$} & \multirow{2}{*}{ Identificación } & \multicolumn{3}{|c|}{$\begin{array}{l}\text { Mediciones de rugosidad } \\
\text { superficial } \mathrm{Ra}(\mu \mathrm{m})\end{array}$} & \multirow{2}{*}{$\begin{array}{l}\text { Promedio } \\
\qquad(\mu \mathrm{m})\end{array}$} & \multirow{2}{*}{$\begin{array}{c}\text { Desviación } \\
\text { estándar } \\
(\mu \mathrm{m})\end{array}$} & \multirow{2}{*}{$\begin{array}{c}\text { Coeficiente } \\
\text { de variación } \\
(\%)\end{array}$} & \multirow{2}{*}{$\begin{array}{c}\text { Sesgo } \\
\text { estandarizado }\end{array}$} & \multirow{2}{*}{$\begin{array}{c}\text { Curtosis } \\
\text { estandarizada }\end{array}$} \\
\hline & & $\begin{array}{c}\text { Medición } \\
1\end{array}$ & $\begin{array}{c}\text { Réplica } \\
1\end{array}$ & $\begin{array}{l}\text { Réplica } \\
\quad 2\end{array}$ & & & & & \\
\hline \multirow{10}{*}{0} & M 2-1 & 20,50 & 15,99 & 15,89 & \multirow{10}{*}{21,1807} & \multirow{10}{*}{3,2874} & \multirow{10}{*}{15,5208} & \multirow{10}{*}{0,3667} & \multirow{10}{*}{$-0,8103$} \\
\hline & M 2-2 & 19,09 & 22,22 & 21,44 & & & & & \\
\hline & M 2-3 & 18,62 & 20,71 & 18,58 & & & & & \\
\hline & M 2-4 & 20,03 & 25,02 & 23,23 & & & & & \\
\hline & M 2-5 & 22,01 & 24,08 & 25,02 & & & & & \\
\hline & M 2-6 & 18,43 & 22,48 & 22,57 & & & & & \\
\hline & M 2-7 & 25,96 & 25,21 & 27,47 & & & & & \\
\hline & M 2-8 & 19,99 & 19,69 & 21,95 & & & & & \\
\hline & M 2-9 & 16,56 & 27,21 & 22,40 & & & & & \\
\hline & M 2-10 & 17,89 & 16,01 & 19,17 & & & & & \\
\hline \multirow{10}{*}{120} & M 2-1 & 25,85 & 26,79 & 18,88 & \multirow{10}{*}{23,6923} & \multirow{10}{*}{3,6065} & \multirow{10}{*}{15,2224} & \multirow{10}{*}{0,2683} & \multirow{10}{*}{$-0,4132$} \\
\hline & M 2-2 & 19,90 & 20,81 & 24,82 & & & & & \\
\hline & M 2-3 & 16,07 & 26,71 & 25,58 & & & & & \\
\hline & M 2-4 & 30,53 & 26,32 & 25,01 & & & & & \\
\hline & M 2-5 & 26,33 & 23,05 & 26,87 & & & & & \\
\hline & M 2-6 & 19,03 & 21,41 & 24,21 & & & & & \\
\hline & M 2-7 & 19,75 & 26,40 & 29,12 & & & & & \\
\hline & M 2-8 & 20,32 & 20,50 & 24,05 & & & & & \\
\hline & M 2-9 & 31,15 & 21,68 & 21,38 & & & & & \\
\hline & M 2-10 & 21,99 & 22,03 & 24,23 & & & & & \\
\hline \multirow{10}{*}{240} & M 2-1 & 22,58 & 18,16 & 38,80 & \multirow{10}{*}{27,5690} & \multirow{10}{*}{4,0901} & & & \\
\hline & M 2-2 & 22,44 & 23,59 & 23,31 & & & & & \\
\hline & M 2-3 & 27,76 & 29,67 & 27,07 & & & & & \\
\hline & M 2-4 & 28,24 & 25,25 & 27,58 & & & & & \\
\hline & M 2-5 & 28,32 & 29,36 & 30,33 & & & 148358 & ( 7472 & 11803 \\
\hline & M 2-6 & 27,25 & 26,38 & 27,03 & & & 14,8358 & $0, / 4 / 2$ & 1,1000 \\
\hline & M 2-7 & 31,36 & 33,40 & 31,50 & & & & & \\
\hline & M 2-8 & 27,15 & 26,68 & 25,77 & & & & & \\
\hline & M 2-9 & 32,11 & 32,00 & 31,58 & & & & & \\
\hline & M 2-10 & 23,26 & 25,14 & 24,00 & & & & & \\
\hline & M 2-1 & 36,06 & 29,55 & 30,76 & & & & & \\
\hline & M 2-2 & 23,49 & 25,04 & 21,98 & & & & & \\
\hline & M 2-3 & 24,56 & 29,93 & 32,81 & & & & & \\
\hline & M 2-4 & 29,92 & 28,22 & 28,36 & & & & & \\
\hline 360 & M 2-5 & 38,89 & 30,61 & 24,54 & 310787 & 51822 & 166744 & 08030 & 06752 \\
\hline 300 & M 2-6 & 29,94 & 28,57 & 25,88 & $31,0 / 8 /$ & 5,1822 & $10,0 / 44$ & 0,8039 & $0,0 / 32$ \\
\hline & M 2-7 & 32,31 & 40,44 & 38,77 & & & & & \\
\hline & M 2-8 & 29,40 & 30,24 & 27,36 & & & & & \\
\hline & M 2-9 & 40,93 & 38,55 & 37,88 & & & & & \\
\hline & M 2-10 & 33,42 & 32,48 & 31,47 & & & & & \\
\hline
\end{tabular}

En la figura 2, se observa que se produce un incremento de la rugosidad a medida que transcurre el tiempo de exposición y los valores de rugosidad en condiciones de exposición exterior son superiores a los obtenidos en condiciones de exposición interior.
Dichos resultados, se demuestran a partir de un análisis estadístico de las medias y desviaciones estándares en la tabla 1 y 2. Las probetas fueron identificadas con la nomenclatura siguiente: condiciones de ensayo exterior (M1) y condiciones de ensayo interior (M2). 


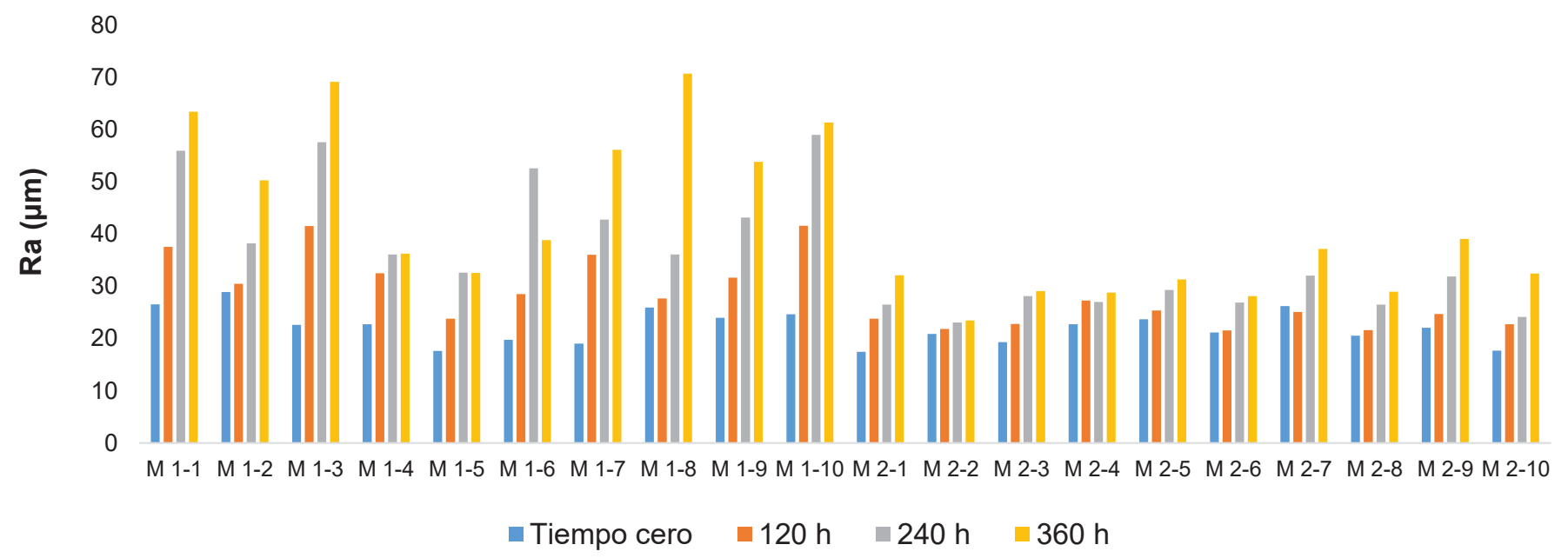

Figura 2. Comportamiento de la rugosidad superficial del acero en condiciones de exposición exterior (M1) e interior (M2), durante un tiempo de $360 \mathrm{~h}$.

Las muestras de acero expuestas a ambiente interior no escapan de la ocurrencia de la corrosión y del aumento de la rugosidad superficial, pero el fenómeno se reduce notablemente, debido a la menor incidencia de factores, como las lluvias, la humedad y los contaminantes atmosféricos. La figura 1 muestra el estado de las probetas ensayadas al inicio del estudio y, una vez finalizado el mismo, en las diferentes condiciones de exposición.

\section{Análisis estadístico}

Diseño multifactorial. El procedimiento utilizado, se basa en un análisis multifactorial, donde se definen dos factores tiempo de exposición y condiciones de exposición a distintos niveles, para la determinación de la variable respuesta, rugosidad superficial. En el diseño programado, se identifica cada factor con los niveles siguientes:

- Tiempo de exposición: 0 h, 120 h, 240 h, 360 h.

- Condiciones de ensayo: Exterior, interior.

- Medición: 1, réplicas: 2 (para cada probeta analizada)

La tabla 3 resume la ANOVA, la cual, particiona la variabilidad de la rugosidad superficial, según la influencia de cada efecto por separado. En este caso, las condiciones de exposición y el tiempo de ensayo presentan un valor-P menor que 0,05 , indicando que ambos factores tienen un efecto estadísticamente significativo, donde al menos una de las medias es diferente, con un nivel de confianza del 95\%. En la figura 3, se observa la existencia de diferentes grupos homogéneos a partir de la prueba LSD de Fisher, con un $95 \%$, donde las medias son estadísticamente diferentes entre las condiciones de exposición en exteriores e interiores.

Ajuste del modelo matemático. Se obtuvieron las ecuaciones del modelo lineal simple, diseñado para construir un modelo estadístico, que describa el impacto de un solo factor cuantitativo $\mathrm{X}$ sobre una variable dependiente $\mathrm{Y}$. En este caso, se describe la relación entre la rugosidad superficial $(\mu \mathrm{m})$ y el tiempo de exposición (h).

- Para condiciones de exposición en exteriores:

$\mathrm{Ra}$ (Micras) exteriores $=23,388+0,085525 *$ Tiempo de exposición (h)

Resultados obtenidos por el programa Statgraphics Centurion XVII Coeficiente de Correlación $=0,99677$

R-cuadrada $=99,3551$ porciento

Tabla 3. Análisis de Varianza para Ra - Suma de Cuadrados Tipo III.

\begin{tabular}{|l|c|c|c|c|c|}
\hline \multicolumn{1}{|c|}{ Fuente } & $\begin{array}{c}\text { Suma de Cuad- } \\
\text { rados }\end{array}$ & Gl & $\begin{array}{c}\text { Cuadrado Me- } \\
\text { dio }\end{array}$ & Razón-F & Valor-P \\
\hline Efectos principales & & & & & \\
\hline A:Condiciones de exposición & 9987,57 & 1 & 9987,57 & 136,38 & 0,0000 \\
\hline B:Tiempo de exposición & 13969,8 & 3 & 4656,61 & 63,59 & 0,0000 \\
\hline Residuos & 17209,7 & 235 & 73,2328 & & \\
\hline Total (corregido) & 41167,1 & 239 & & & \\
\hline
\end{tabular}




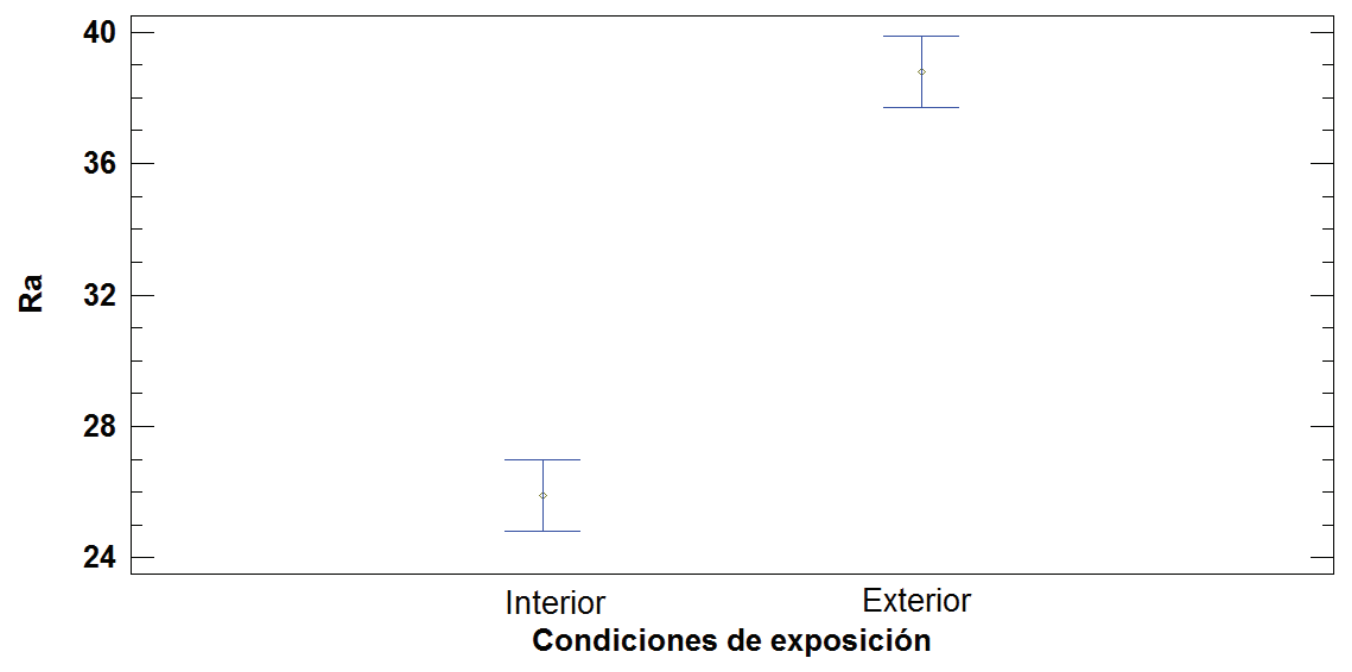

Figura 3. Análisis de las medias, según la prueba LSD de Fisher 95\%.

R-cuadrado (ajustado para g.l.) $=99,0327$ porciento

Error estándar del est. $=1,30733$

Error absoluto medio $=0,773$

Estadístico Durbin-Watson $=2,94414(\mathrm{P}=0,6612)$

Autocorrelación de residuos en retraso $1=-0,587193$

El estadístico R-Cuadrada indica que el modelo ajustado explica $99,3551 \%$ de la variabilidad en Ra (Micras) en condiciones de exposición en exteriores. El coeficiente de correlación es igual a 0,99677 , indicando una relación relativamente fuerte entre las variables. El 0,6449\% es atribuible a las desviaciones alrededor de la línea, las cuales, pueden ser por otros factores, como el error de medición o un error del modelo lineal, para ajustar los datos adecuadamente.

El error estándar del estimado indica que la desviación estándar de los residuos es 1,30733. Este valor, se puede usar para construir límites de predicción para nuevas observaciones.

El error absoluto medio (MAE) de 0,773 es el valor promedio de los residuos. El estadístico de Durbin-Watson (DW) examina los residuos para determinar si hay alguna correlación significativa basada en el orden en el que se presentan en el archivo de datos. Puesto que el valor-P es mayor que 0,05 , no hay indicación de una autocorrelación serial en los residuos con un nivel de confianza del $95,0 \%$

- Para condiciones de exposición en interiores:

Ra (Micras) Interiores $=20,843+0,0279833 *$ Tiempo de exposición (h)

Resultados obtenidos por el programa Statgraphics Centurion XVII

Coeficiente de Correlación $=0,996459$

R-cuadrada $=99,2931$ porciento

R-cuadrado (ajustado para g.l.) $=98,9397$ porciento
Error estándar del est. $=0,447984$

Error absoluto medio $=0,2555$

Estadístico Durbin-Watson $=2,52801(\mathrm{P}=0,3479)$

Autocorrelación de residuos en retraso $1=-0,438574$

El estadístico R-Cuadrada indica que el modelo ajustado explica 99,2931\% de la variabilidad en Ra (Micras) en condiciones de exposición de interiores. El coeficiente de correlación es igual a 0,996459 , indicando una relación relativamente fuerte entre las variables. El 0,7069\% es atribuible a las desviaciones alrededor de la línea, las cuales, pueden ser por otros factores, como el error de medición o un error del modelo lineal, para ajustar los datos adecuadamente.

El error estándar del estimado indica que la desviación estándar de los residuos es 0,447984. Este valor, se puede usar para construir límites de predicción para nuevas observaciones.

El error absoluto medio (MAE) de 0,2555 es el valor promedio de los residuos. El estadístico de Durbin-Watson (DW) examina los residuos para determinar si hay alguna correlación significativa basada en el orden en el que se presentan en el archivo de datos. Puesto que el valor-P es mayor que 0,05 , no hay indicación de una autocorrelación serial en los residuos con un nivel de confianza del $95,0 \%$.

La pendiente obtenida en la ecuación del modelo lineal simple Ra vs. Tiempo en condiciones exteriores resulta superior al valor de la pendiente en condiciones interiores, demostrando la diferencia entre los resultados.

Estadígrafos obtenidos. Teniendo en cuenta los valores obtenidos de la rugosidad superficial reportados en la tabla 1 y 2 , se calcularon los estadígrafos correspondientes a valores promedios, desviación 
estándar, curtosis estandarizada y sesgo estandarizado. Se puede evidenciar que la tasa de crecimiento de las medias en el exterior es superior a las condiciones de exposición en interior. En ambos casos, los valores del sesgo estandarizado y la curtosis estandarizada, se encuentran dentro del rango esperado, de -2 a 2 , indicando que los datos provienen de una distribución normal.

El presente estudio permitió determinar:

- Las variables tiempo de exposición y condiciones de ensayo seleccionadas en el análisis factorial presentan un efecto estadísticamente significativo en los valores de rugosidad superficial, con un nivel de confianza del 95\%. Los valores de las medias obtenidos para condiciones de exposición en exterior e interior pertenecen a grupos homogéneos diferentes, calculados a partir de la prueba LSD de Ficher.

- Los estadígrafos sesgo estandarizado y curtosis estandarizada, obtenidos en las mediciones de rugosidad superficial en ambas condiciones de exposición, se encontraban en el rango esperado, indicando una distribución normal.

- La pendiente de los modelos lineales obtenidos indica el aumento de la rugosidad superficial, a medida que transcurre el tiempo de exposición en ambos medios de exposición.

- Las ecuaciones del modelo lineal simple obtenidas describen la relación entre la rugosidad superficial y el tiempo de exposición para condiciones exteriores e interiores. Las mismas indican una relación relativamente fuerte entre las variables y la no existencia de una autocorrelación serial en los residuos, con un nivel de confianza del 95,0\%, posibilitando ciertas predicciones en el futuro, para condiciones del clima similares a las descritas en el estudio, para tiempos cortos.

Conflicto de intereses: El manuscrito fue preparado y revisado exclusivamente por los autores, quienes declaran no tener conflicto de interés que ponga en riesgo la validez de los resultados aquí presentados.

\section{REFERENCIAS}

1. AHMAD, Z. 2006. Principles of corrosion engineering and corrosion control. Elsevier Ltd. 672p. https://doi. org/10.1016/B978-0-7506-5924-6.X5000-4

2. CAMEJO GINIEBRA, J.J.; DOMÍNGUEZ DOMÍNGUEZ, J.; MARRERO ÁGUILA, R. 2018. Apuntes sobre corrosión y protección contra la corrosión. Reporte Técnico. U. Tecnológica de la Habana, José Antonio Echeverría. 114p.

3. CASTAÑO, J.; BOTERO, C.; PEÑARANDA, S. 2007. Corrosión atmosférica del zinc en ambientes exteriores e interiores. Rev. Metalúrgica. (España). 43(2):133-145.

4. CHICO, B.; DE LA FUENTE, D.; MORCILLO, M. 2000.
Corrosión atmosférica de metales en condiciones climáticas extrema. Bol. Sociedad Española de Cerámica y Vidrio. 39(3):309-332.

5. DEL ANGEL-MERAZ, E.; VELEVA, L.; ACOSTAALEJANDRO, M. 2009. Agresividad atmosférica basada en el tiempo de humectación del clima tropical húmedo del estado de Tabasco. Universidad y Ciencia Trópico Húmedo. (México). 25(2):111-120.

6. DOMÍNGUEZ DOMÍNGUEZ, J.; CASTRO, D.M. 1987. Introducción a la corrosión y la protección de metales. Cuba: Ministerio de Educación Superior

7. GONZÁLEZ PRADA, C.; MARRERO ÁGUILA, R. 2005. Factores ambientales y su influencia en la agresividad corrosiva de la estación de ensayos de Cojímar. Comparación con otras estaciones. Rev. CENIC. Ciencias Químicas. (Cuba). 36(3):181-185.

8. GUERRA, J.C.; CASTAÑEDA, A.; CORVO, F.; HOWLAND, J.; RODRÍGUEZ, J. 2018. Atmospheric corrosion of low carbon steel in a coastal zone of Ecuador: Anomalous behavior of chloride deposition versus distance from the sea. Materials and Corrosion. (Alemania). 2018:1-17. https://doi.org/10.1002/maco.201810442

9. HONG, T.; NAGUMO, M. 1997. Effect of surface roughness on early stages of pitting corrosion of type 301 stainless steel. Corrosion Science. 39(9):1665-1672. https://doi. org/10.1016/S0010-938X(97)00072-3

10. INTERNATIONAL ORGANIZATION FOR STANDARDIZATION, ISO. 1992. ISO 9226: Corrosion of metals and alloys - Corrosivity of atmospheres Determination of corrosion rate of standard specimens for the evaluation of corrosivity

11. INTERNATIONAL ORGANIZATION FOR STANDARDIZATION, ISO. 2012. ISO 9223: Corrosion of metals and alloys - Corrosivity of atmospheres Classification, determination and estimation. 15p.

12. LIU, C.; LEYLAND, A.; LYON, S.; MATTHEWS, A. 1995. An a.c. impedance study on PVD CrN-coated mild steel with different surface roughnesses. Surface and Coating Technology. 1:623-631. https://doi.org/10.1016/02578972(95)02544-8

13. LÓPEZ CABRERA, C.M.; ITURRALDE VINENT, M.A.; CLARO MADRUGA, R.; RUIZ GUTIÉRREZ, L.; CABRERA TRIMIÑO, G.J.; MOLERIO LEÓN, L.; CHAMIZO LARA, A.R.; GARCÍA SUÁREZ, L.; GERHARTZ MURO, J.L.; GARCÍA MONTERO, G.; PEREZ CAO, H.; PINO RODRÍGUEZ, A.; SENTÍ DARIAS, M.M.; BORROTO PONCE, R.; RODRÍGUEZ 
CARRILLO, Y. 2006. Introducción al conocimiento del medio ambiente Vol. 1. Editorial-Académica. 32p.

14. MOAYED, M.H.; LAYCOCK, N.J.; NEWMAN, R.C. 2003. Dependence of the critical pitting temperature on surface roughness. Corrosion Science. 45:1203-1121. https://doi. org/10.1016/S0010-938X(02)00215-9

15. MOK LEE, S.; GYU LEE, W.; HO KIM, Y.; HO JANG, Y. 2012. Surface roughness and the corrosion resistance of $21 \mathrm{Cr}$ ferritic stainless steel. Corrosion Science. 63:404-409. https://doi.org/10.1016/j.corsci.2012.06.031

16. NOR ASMA, R.B.A.; YULI, P.A.; MOKHTAR, C.I. 2011. Study on the effect of surface finish on corrosion of carbon steel in CO2. J. Applied Sciences. (Pakistan). 11(11):2053-2057. http:/ / doi.org/10.3923/jas.2011.2053.2057

17. OFICINA NACIONAL DE NORMALIZACIÓN, ONN. 1981. NC 16-60. Normas básicas de intercambiabilidad. Rugosidad superficial. Párametros principales y valores numéricos. $4 \mathrm{p}$.

18. RODRÍGUEZ GRASSO, J.M.; TORRES VALHUERDI, A.; ALONSO GONZÁLEZ, A. 2008. Rugosidad Superficial. $\mathrm{CD}$ de Monografías. Universidad de Matanzas "Camilo Cienfuegos". 22p.
19. SÁNCHEZ MUÑOZ, M. 2017. Análisis de Riesgos Industriales en Plantas Químicas y Petroleras. Método HAZ-OP (XVII Edición). 179p.

20. SASAKI, K.; BURSTEIN, G.T. 1996. The generation of surface roughness during slurry erosion-corrosion and its effect on the pitting potential. Corrosion Science. 38(12):2111-2120. https://doi.org/10.1016/S0010-938X(96)00066-2

21. SUÁREZ CORRALES, X.I.; CORVO PÉREZ, F.E.; VILLAR LÓPEZ, R.V.; MARRERO ÁGUILA, R. 2014. Resistencia al clima tropical de aceros galvanizados con y sin recubrimiento. Ingeniería, Investigación y Tecnología. (México). 15(1):29-40. https://doi.org/10.1016/S14057743(15)30004-4

22. TEJERO RIVAS, M.C.; BAUTISTA MARGULIS, R.G.; DEL ÁNGEL MERAZ, E.; HERNÁNDEZ MORALES, N.E. 2015. Corrosividad atmosférica del cobre y del acero en dos localidades de Villahermosa, Tabasco. Ingeniería Investigación y Tecnología. 16(2):197-206. https://doi. org/10.1016/j.riit.2015.03.004 